\title{
PROFILO EPIDEMIOLOGICO E DETERMINANTE SOCIALE DEL COVID-19 A MACAPÁ, AMAPÁ, AMAZON, BRASILE
}

\section{ARTICOLO ORIGINALE}

SILVA, Anderson Walter Costa $^{1}$, CUNHA, Arthur Arantes ${ }^{2}$, ALVES, Giovana Carvalho ${ }^{3}$, CORONA, Rodolfo Antônio ${ }^{4}$, DIAS, Claudio Alberto Gellis de Mattos ${ }^{5}$, NASSIRI, Reza ${ }^{6}$, VEDOVELLI, Silvana7, VILHENA, Tania Regina Ferreira ${ }^{8}$, FAVACHO, Veronica Batista Cambraia ${ }^{9}$, SOUSA, Josiany Ferreira ${ }^{10}$, ARAÚJO, Maria Helena Mendonça ${ }^{11}$, OLIVEIRA, Euzébio ${ }^{12}$, DENDASCK, Carla Viana ${ }^{13}$, FECURY, Amanda Alves ${ }^{14}$

\footnotetext{
${ }^{1}$ Médico, Especialista em Gestão de Sistemas e Serviços de Saúde. Professore, precettore e ricercatore del Corso di Medicina del Campus Macapá dell'Università Federale di Amapá (UNIFAP).

${ }^{2}$ Accademico del Corso di Medicina del Campus Macapá, Università Federale di Amapá (UNIFAP).

${ }^{3}$ Accademico del Corso di Medicina del Campus Macapá, Università Federale di Amapá (UNIFAP).

${ }^{4}$ Accademico del Corso di Medicina del Campus Macapá, Università Federale di Amapá (UNIFAP).

${ }^{5}$ Biologo, Dottore di Ricerca in Teoria e Comportamento, Professore e ricercatore del Corso di Laurea in Chimica dell'Istituto di Educazione Di Base, Tecnica e Tecnologica di Amapá (IFAP).

${ }^{6}$ Medico. Dottorato di ricerca in Ematologia, Specialista in Farmacologia Clinica con esperienza in salute globale e Infezioni Virali. Professore del Dipartimento di Farmacologia e Tossicologia, della Medicina familiare e comunitaria. Università statale del Michigan (MSU), Michigan, USA.

${ }^{7}$ Infermiera. Segretario sanitario del comune di Macapá AP (SESA AP).

8 Infermiera. Master in Scienze della Salute. Dipartimento sanitario comunale di Amapá (SESA AP).

${ }^{9}$ Infermiera. Dottorato di ricerca in Scienze. Dipartimento sanitario comunale di Amapá (SESA AP).

${ }^{10}$ Infermiera. Dipartimento sanitario comunale di Amapá (SESA AP).

${ }^{11}$ Dottore, Master in Scienze dell'Insegnamento e della Salute, Professore, precettore e ricercatore del Corso di Medicina del Campus Macapá, Università Federale di Amapá (UNIFAP).

${ }^{12}$ Biologo, Dottore di Ricerca in Malattie Topiche, Professore e ricercatore del Corso di Educazione Fisica, Università Federale di Pará (UFPA).

${ }^{13}$ Teologo, Dottore di Ricerca in Psicoanalisi, ricercatore presso il Centro di Ricerca e Studi Avanzati - CEPA.

${ }^{14}$ Biomedicale, Dottorato di Ricerca in Malattie Topiche, Professore e ricercatore del Corso di Medicina del Campus Macapá, Università Federale di Amapá (UNIFAP).
}

RC: 71075

Disponibile in: https://www.nucleodoconhecimento.com.br/salute/del-covid-19-a$\underline{\text { macapa }}$ 
SILVA, Anderson Walter Costa. Et. Profilo epidemiologico e determinante sociale del COVID-19 a Macapá, Amapá, Amazon, Brasile. Revista Científica Multidisciplinar Núcleo do Conhecimento. Anno 05, Ed. 04, Vol. 04, pp. 05-27. nell'aprile 2020. ISSN: 2448-0959, Link di accesso: https://www.nucleodoconhecimento.com.br/salute/del-covid-19-a-macapa, $\quad$ DOI: 10.32749/nucleodoconhecimento.com.br/salute/del-covid-19-a-macapa

\section{RIEPILOGO}

Alla fine di dicembre 2019, a Wuhan, in Cina, è emersa una nuova varietà di Coronavirus, dichiarata emergenza sanitaria pubblica globale dall'Organizzazione Mondiale della Sanità (OMS). II tropismo delle vie aeree può essere dovuto all'espressione dell'enzima angiotensina-convertitore 2 (ACE2). Nella regione dell'Amazzonia legale brasiliana, fino al 22 marzo 2020, lo Stato di Amazonas ha presentato 26 casi confermati; Acri 11 casi; Paragrafo 04; Rondonia 03; Roraima, Tocantins, Maranhão e Mato Grosso 02 casi; e Amapá solo 01 se confermato. Questo studio mira a quantificare e analizzare i primi casi sospetti e confermati di COVID-19 a Macapá, Amapá, Amazon, Brasile. Si tratta di uno studio osservazionale, retrospettivo e quantitativo, riferito al profilo dei primi 108 casi sospetti segnalati a Macapá, tra il 13 marzo 2020 e il 21 marzo 2020, e ai calcoli di incidenza del COVID-19 nelle 26 capitali brasiliane e Brasilia, Distretto Federale, tra il 26 febbraio 2020 e il 26 marzo 2020. Gli studi possono rivelare un modello specifico di diffusione del virus, che contribuirebbe alla pianificazione e all'attuazione di misure di controllo e sorveglianza epidemiologica più efficaci.

Parole chiave: COVID 19, ACE2, coronavirus, pandemia.

\section{INTRODUZIONE}

Alla fine di dicembre 2019, a Wuhan, in Cina, è emersa una nuova varietà di Coronavirus, in grado di produrre sindrome respiratoria acuta grave (SRHS),

$\mathrm{RC}: 71075$

Disponibile in: https://www.nucleodoconhecimento.com.br/salute/del-covid-19-amacapa 
dichiarata un'emergenza sanitaria pubblica globale dall'Organizzazione Mondiale della Sanità. Questo virus, chiamato SARS-CoV-2, produce una malattia classificata come COVID-19 (ICD-10-B34.2) (BRASIL, 2020a; OMS, 2020a; MCINTOSH, 2020). SARS-CoV-2 appartiene al genere betaCovs, della sottofamiglia Orthocoronavirinae, della famiglia Coronaviridae. È un virus envelopado (una doppia membrana lipidica con proteine inserite in esso) di RNA a nastro singolo, che ha la forma di una corona, a causa delle glicoproteine a forma spinosa presenti nel suo involucro (CASCELLA et al., 2020; VELAVAN et al., 2020).

I sintomi più comuni ma non specifici legati all'infezione da questo virus sono: febbre $(83,0 \%-99,0 \%)$, tosse secca $(59,4 \%-82,0 \%)$, dispnea (55,0\%), affaticamento (stanchezza) (38,1\%), mal di gola (13,9\%), mal di testa (mal di testa) $(13,6 \%)$ e diarrea (3,7\%) (BRASIL, 2020a; LI et al., 2020; OMS, 2020a). Questi sintomi possono apparire in linea con il tempo di incubazione del virus, in media, da 5 a 6 giorni dopo l'infezione (OMS, 2020a; ROTHAN et al., 2020).

Il tropismo delle vie aeree può essere dovuto all'espressione dell'enzima convertitore di angiotensina 2 (ACE2) nel parenchima polmonare, nell'epitelio delle vie aeree umane e nell'endotelio vascolare. ACE2 è uno dei principali mediatori dell'ingresso del virus nelle cellule dell'ospite umano, fungendo da recettore per l'ingresso di agenti patogeni. Tuttavia, questo meccanismo non è sufficiente a spiegare il coinvolgimento delle linee cellulari umane, che non esprimevano ACE2 ma erano infette (LI et al., 2020; LI et al., 2003; ROTHAN et al., 2020).

Una caratteristica della SARS-CoV-2, che ha contribuito a diventare un problema globale di salute pubblica, è il suo alto potenziale di velocità / trasmissione, che si verifica attraverso la femite (materiali inanimati contaminati che fungono da veicolo per la trasmissione) e goccioline respiratorie di tosse e / o starnuti (trasmissione aerosol). Questa trasmissione può avvenire, a seconda della concentrazione di particelle virali nell'ambiente, e la vitalità virale nella dispersione di aerosol è stata dimostrata per tre ore o più. La via orofecale non sembra efficace, anche se in alcuni

RC: 71075

Disponibile in: https://www.nucleodoconhecimento.com.br/salute/del-covid-19-amacapa 
casi sono stati trovati virus vitali (CASCELLA, et al., 2020; DOREMALEN, et al., 2020; WHO, 2020a). SARS-Cov-2 è la terza minaccia globale per la salute pubblica dopo SARS e MERS (NASSIRI, 2020).

Secondo il Centro operativo per le emergenze di sanità pubblica (COE-nCoV) le misure di prevenzione di base sono: lavarsi frequentemente le mani usando acqua e sapone (per almeno 20 secondi); nell'impossibilità di utilizzare acqua e sapone, utilizzare come alcol alternativo il $70 \%$ o un disinfettante a base alcolica; evitare tocchi su viso, occhi e bocca con le mani non lavati; coprire con la bocca e il naso del fazzoletto quando starnutisce o tossisce (e poi scartare la sciarpa); pulire oggetti e superfici frequentemente toccati (maniglia della porta, cellulare, telecomando); evitare il contatto con persone malate (astinenza); e rimanere a casa se si presentano sintomi, anche se l'influenza comune (BRASIL, 2020b).

L'avvio del protocollo per il trattamento della polmonite da COVID (2019-nCoV) dipende dal riconoscimento precoce dei segni, monitorati continuamente, tenendo conto delle manifestazioni cliniche e delle caratteristiche generali dell'infezione. Per questo, il Ministero della Salute brasiliano ha creato un protocollo di trattamento (BRASIL, 2020b; BRASIL, 2020c).

L'elevato tasso/potenziale di trasmissione, combinato con l'attuale mancanza di un farmaco antivirale specifico per il trattamento (BRASIL, 2020c), ha fornito i 55.924 casi di COVID-19 confermati in Cina, fino al 20 febbraio 2020 (OMS, 2020b). Letalità di $44.672(79,9 \%)$ varia a seconda della fascia d'età, essendo lo $0,2 \%$ nei pazienti di età compresa tra 10 e 39 anni e raggiungendo l'8,0\% nei pazienti di età compresa tra 70 e 79 anni e il 14,8\% nei pazienti di età compresa tra $\geq 80$ anni, il gruppo più vulnerabile. La variazione del tasso di letalità può essere giustificata da fattori di rischio associati, come la precedente storia delle malattie cardiovascolari, il diabete, l'ipertensione, le malattie respiratorie croniche e il cancro (GALLASCH et al., 2020; ZHANG et al., 2020; WHO, 2020a). In Italia, il secondo Paese con il maggior coinvolgimento, fino al 20 marzo 2020, sono stati confermati 53.578 casi di COVID-

$\mathrm{RC}: 71075$

Disponibile in: https://www.nucleodoconhecimento.com.br/salute/del-covid-19-amacapa 
19, con 4.827 decessi, che hanno portato a una letalità generale del 9,0\% (OMS, 2020b).

In Brasile, il primo caso di COVID-19 è stato confermato il 26 febbraio 2020 (OMS, 2020c) e fino al 22 marzo 2020, sono stati confermati 1.546 casi e 25 decessi (22 nello stato di São Paulo e 03 nello stato di Rio de Janeiro) da COVID-19. Di questo totale, $926(59,9 \%)$ sono stati registrati nella regione sud-orientale; 231 (14,9\%) nella regione nord-orientale; $179(11,6 \%)$ nella regione meridionale; $161(10,4 \%)$ nella regione del Midwest; e $49(3,2 \%)$ nella regione settentrionale del paese (BRASIL, 2020d).

Nella regione del Nord, fino alla stessa data, lo Stato amazzonio ha presentato 26 casi confermati; Acri 11 casi; Paragrafo 04; Rondônia 03; Roraima e Tocantins 02 casse; e Amapá solo 01 casi confermati (BRASIL, 2020d).

Dato l'aumento esponenziale dei casi di COVID-19 e l'istituzione di sfide per la salute pubblica brasiliana, gli studi epidemiologici che valutano le specificità della popolazione coinvolta e analizzano l'influenza dei fattori sociali sui tassi di trasmissibilità del virus sono estremamente importanti e di necessità sanitaria (LIPSITCH et al., 2020).

\section{GOL}

Quantificazione e analisi dell'indice iniziale dei casi sospetti e successivamente confermati di COVID-19 a Macapá, Amapá, Amazon, Brasile.

Analizzare l'incidenza dei casi confermati di COVID-19 nelle capitali situate nell'Amazzonia Legale rispetto ad altre capitali brasiliane e correlare con indicatori socioeconomici, sociodemografici e sanitari.

RC: 71075

Disponibile in: https://www.nucleodoconhecimento.com.br/salute/del-covid-19-amacapa 


\section{MATERIALI E METODI}

\section{PERIODI DI STUDIO}

Si tratta di uno studio osservazionale, retrospettivo e quantitativo. Questo studio ha due periodi di tempo. Uno di questi, riferito al profilo dei primi 108 casi sospetti segnalati a Macapá, è limitato tra il 13 marzo 2020, data di notifica del primo caso sospetto in Brasile (OMS, 2020c), e il 21 marzo 2020, a causa dell'ordinanza n. 454, del Ministero della Salute (MS), che ha dichiarato lo stato di trasmissione della comunità in Brasile (BRASIL, 2020e). Si noti che i casi notificati fino al 21 marzo possono avere la loro conferma/smaltimento pochi giorni dopo la notifica, a causa del termine per l'analisi di laboratorio.

L'altro periodo di tempo di questo studio, riferito ai calcoli di incidenza del COVID-19 nelle 26 capitali brasiliane e Brasilia, Distretto Federale, è limitato tra il 26 febbraio 2020, data del primo caso confermato in Brasile (OMS, 2020c), e il 26 marzo 2020, un mese dopo la conferma del primo caso.

\section{DATI, VARIABILI E METODI DI CALCOLO}

Questo studio ha utilizzato dati secondari. I dati relativi al profilo dei primi 108 casi sospetti a Macapá sono stati estratti dal database e dalle informazioni aggregate sviluppate dal Dipartimento della Salute del comune di Macapá, Amapá, Brasile.

I dati sulla popolazione, per intervallo, del comune di Macapá sono stati stimati dall'indagine nazionale per campione di famiglie continue 2016-2018 (BRASIL, 2018a), con riferimento nella popolazione di Macapá nel 2019 (BRASIL, 2020f). Questi dati sulla popolazione sono stati utilizzati nel calcolo del coefficiente del caso sospetto (CcS) per fascia d'età, indipendentemente dal sesso, che è stato calcolato secondo la formula:

RC: 71075

Disponibile in: https://www.nucleodoconhecimento.com.br/salute/del-covid-19-amacapa 
Coeficiente $(\mathrm{CCS})=\frac{\text { Número de casos suspeitos em determinada faixa etária } \times 100.000}{\text { Quantitativo populacional estimado da faixa etária referida }}$

I casi quantitativi confermati di COVID-19, utilizzati per calcolare le incidenze (ogni 100.000 persone) nelle capitali brasiliane, fino al 26 marzo 2020, sono stati estratti dalla Mappa del Coronavirus (2020), che fornisce dati aggregati ufficiali e aggiornati, forniti dai Dipartimenti sanitari statali, di tutti i comuni brasiliani con casi confermati. D'altra parte, i dati sulla popolazione utilizzati nel calcolo delle incidenze sono stati estratti da IBGE - Città e Stati (BRASIL, 2020f). II metodo di calcolo dell'incidenza è stato:

$$
\text { Incidência }=\frac{\text { Número de casos confirmados em determina capital } \times 100.000}{\text { Quantitativo populacional da capital referida, no ano de } 2019}
$$

Gli indicatori socioeconomici dell'indice di sviluppo umano (HDI) e della percentuale di poveri sono stati estratti dalla piattaforma Atlas Brasil (2020). II numero di strutture sanitarie (periodo di riferimento: ottobre 2015) è stato estratto dalla piattaforma DATASUS (BRASIL, 2020g) del Ministero della Salute (SM). La densità demografica (DD) è stata calcolata utilizzando informazioni recenti, poiché l'ultimo funzionario si riferisce al censimento IBGE del 2010. Pertanto, i dati dell'area territoriale della capitale (anno di riferimento: 2018) e della popolazione di capitale (anno di riferimento: 2019) sono stati utilizzati per calcolare DD (DD = popolazione capitale nel 2019 / superficie capitale in chilometri quadrati nel 2018).

\section{CRITERI DI INCLUSIONE ED ESCLUSIONE DEI DATI}

Per l'analisi sessuale dei primi 108 record, sono stati inclusi tutti i casi sospetti $(\mathrm{n}=108)$; il tempo tra il primo segno/sintomo e la notifica del caso, sono stati esclusi otto casi, cinque maschi e tre femmine $(n=100)$; età, paese di residenza, numero di segni/sintomi e morbilità precedenti, è stato escluso un solo caso femminile $(n=107)$;

RC: 71075

Disponibile in: https://www.nucleodoconhecimento.com.br/salute/del-covid-19-amacapa 
per quanto riguarda il viaggio fuori dal Brasile e il contatto con casi sospetti o confermati, sono stati esclusi tre casi, tutti femminili $(n=105)$; per quanto riguarda l'unità sanitaria di notifica, sono stati esclusi 12 casi $(n=96)$; l'occupazione è stata esclusa 3 casi $(n=105)$. Tutte le esclusioni erano dovute alla mancanza di informazioni ("ignorate"). Gli outlier non sono stati esclusi dall'analisi.

Nell'analisi delle incidenze e delle correlazioni, sono state incluse tutte le 26 capitali delle Unità Federative Brasiliane e Brasília, capitale federale. Le capitali sono state divise in due gruppi, uno composto dalle capitali brasiliane situate nella regione brasiliana dell'Amazzonia Legale (Belém, Boa Vista, Cuiabá, Macapá, Manaus, Palmas, Porto Velho, Rio Branco e São Luís) (BRASIL, 2014) e l'altro dalle restanti 17 capitali più la capitale federale. II numero totale di casi confermati fino a 21 ore e 6 minuti il 26 marzo 2020 è stato incluso per calcolare l'incidenza. Gli outlier non sono stati esclusi dall'analisi.

\section{ANALISI STATISTICA}

I dati sono stati organizzati, tabulati e analizzati utilizzando il software Microsoft Excel ${ }^{\circledR} 2016$, il software OriginPro ${ }^{\circledR}$ versione 8.0724 e il pacchetto statistico per il software Social Sciences ${ }^{\circledR}$ versione 20.0. È interessante notare che il software OriginPro ${ }^{\circledR}$ utilizzato non accettava l'inserimento di un accento acuto nella grafica.

La distribuzione dei dati, se del caso, è stata testata utilizzando i test di KolmogorovSmirnov e Shapiro-Wilk. La scelta della prova è stata effettuata in base alla dimensione del campione (TORMAN et al., 2012). L'omogeneità della varianza, se pertinente, è stata testata con il test di Levene a base media (LEVIN et al., 2018).

Per l'analisi statistica dei primi 108 casi sospetti, il test t dello studente è stato utilizzato per distinguere i mezzi di campioni indipendenti o il test Mann-Whitney U per la differenza mediana. Le variabili che non avevano l'ipotesi di distribuzione normale rifiutata e che presentavano omogeneità della varianza sono state testate

$\mathrm{RC}: 71075$

Disponibile in: https://www.nucleodoconhecimento.com.br/salute/del-covid-19-amacapa 
con il test $t$, mentre le variabili che non soddisfacevano questi requisiti sono state testate con il test di Mann-Whitney (TORMAN et al., 2012). Abbiamo analizzato, confrontando i sessi, l'esistenza significativa della differenza di età media o mediana (in anni), l'intervallo di giorni tra il primo segno / sintomo fino alla data di notifica del caso sospetto e il numero quantitativo di segni / sintomi presentati. II livello di significatività era $p$-value $\leq 0,05$.

L'analisi della differenza di incidenza tra le capitali situate nella regione brasiliana dell'Amazzonia legale $(n=9)$ e le altre capitali brasiliane $(n=18)$ è stata effettuata senza tenere conto dell'ipotesi di una distribuzione normale, a causa della dimensione del campione inferiore a 10. Così, è stata eseguita l'alternativa non parametrica dell'analisi, il test mann-whitney u. II livello di significatività era $p$-value $\leq$ 0,05 (LEVIN et al., 2018; TORMAN et al., 2012).

L'ipotesi di una distribuzione normale non è stata respinta per le seguenti variabili: incidenza delle capitali brasiliane, HDI delle capitali brasiliane, percentuali di poveri nelle capitali brasiliane e quantitativo delle strutture sanitarie nelle capitali brasiliane. Così, il test di correlazione di Pearson (bivariato) è stato continuato. Sono state osservate le direzioni delle correlazioni (positive o negative) e l'intensità del coefficiente di correlazione di Pearson (r). L'intensità (forte, moderata, debole o inesistente) viene valutata in base al valore $r$ (LEVIN et al., 2018). II livello di significatività adottato è stato di valore $p \leq 0,05$.

Sono state calcolate statistiche descrittive: media $(\bar{x})$, deviazione standard, mediana, minima e massima.

\section{LIMITAZIONI}

Tra i limiti di questo studio ci sono casi che non dovrebbero essere segnalati come sospetti, secondo le linee guida della MS (BRASIL, 2020h), ma che co compongono il campione studiato. E l'assenza di alcune informazioni nel database (come

RC: 71075

Disponibile in: https://www.nucleodoconhecimento.com.br/salute/del-covid-19-amacapa 
professione, data del primo sintomo e unità sanitaria di notifica), probabilmente a causa di un completamento errato o incompleto del modulo di notifica.

\section{ETHICAL ASPECTS}

Lo studio ha utilizzato dati secondari aggregati, che non consentono l'identificazione individuale, disponibili nel database del Dipartimento Sanitario Comunale di Macapá, Amapá, Amazon, Brasile, dopo un accordo istituzionale. Oltre alle informazioni disponibili presso gli indirizzi elettronici ad accesso aperto. Questa ricerca ha seguito i criteri delle risoluzioni numero 466/2012 e numero 510/2016 del Consiglio nazionale brasiliano per l'etica della ricerca (CONEP).

\section{RISULTATI}

\section{ANALISI DEI PRIMI 108 CASI SOSPETTI A MACAPÁ}

Dei 108 casi sospetti analizzati in questo studio, segnalati tra il 13 marzo 2020 e il 21 marzo 2020 , tre $(2,78 \%)$ sono stati confermati dall'analisi delle molecole (RT PCR) con COVID-19. Tutti erano brasiliani, due casi femminili (66,66\%) e uno dei maschi $(33,33 \%)$. L'età media dei tre casi era di 36,3 anni. I tre (100,0\%) febbre, mal di testa, naso che cola. Due $(66,66 \%)$ casi avevano tosse, produzione di espettorato e difficoltà respiratorie. Tutti e tre hanno fatto un viaggio fuori dal Brasile nei 14 giorni precedenti la data di notifica. L'ora media dei tre casi confermati tra la data del primo segno/sintomo e la data di notifica è stata di 3 giorni.

II profilo del numero totale di casi sospetti analizzati è stato il seguente: $(n=107$; $99,07 \%)$, femmina ( $n=59 ; 54,62 \%)$, fascia d'età $30-39$ anni $(n=29 ; 27,1 \%)$, senza morbilità precedenti $(n=78 ; 72,22 \%)$ (rispettivamente tabella 1 e tabella 2$)$. L'età complessiva media (compresi entrambi i sessi) negli anni è stata di $\bar{x}=34,2 \pm 14,9$, con una mediana di 34 anni. Non vi è stata alcuna differenza significativa nella media tra i sessi $(t=0,331 ; p=0,742)$ (tabella 3$)$.

RC: 71075

Disponibile in: https://www.nucleodoconhecimento.com.br/salute/del-covid-19-amacapa 
Tabella 1 Frequenza e percentuale, per sesso e fascia d'età, dei casi sospetti di COVID-19 analizzati e Coefficiente per fascia d'età. Macapá, Amapá, 13-21 marzo 2020.

\begin{tabular}{|c|c|c|c|c|c|c|}
\hline \multirow{2}{*}{$\begin{array}{c}\text { Faixa Etária } \\
\text { (anos) }\end{array}$} & \multirow{2}{*}{$\begin{array}{c}\text { Masculino } \\
\text { n (\%) }\end{array}$} & \multirow{2}{*}{$\begin{array}{c}\text { Feminino } \\
\mathbf{n}(\%)\end{array}$} & \multicolumn{2}{|c|}{ Total } & \multirow{2}{*}{$\begin{array}{c}\text { *População } \\
\text { Estimada }\end{array}$} & \multirow{2}{*}{$\begin{array}{c}* * \text { Coeficiente } \\
\text { (por } 100.000 \text { pessoas) }\end{array}$} \\
\hline & & & $\mathbf{n}$ & $\%$ & & \\
\hline $0-19$ & $7(54 \%)$ & $6(46 \%)$ & 13 & $12,1 \%$ & 174.395 & 7,45 \\
\hline $20-29$ & $11(41 \%)$ & $16(59 \%)$ & 27 & $25,2 \%$ & 90.708 & 29,77 \\
\hline $30-39$ & $17(59 \%)$ & $12(41 \%)$ & 29 & $27,1 \%$ & 74.386 & 38,99 \\
\hline $40-49$ & $7(30 \%)$ & $16(70 \%)$ & 23 & $21,5 \%$ & 73.120 & 31,46 \\
\hline $50-59$ & $4(40 \%)$ & $6(60 \%)$ & 10 & $9,3 \%$ & 42.460 & 23,55 \\
\hline$\geq 60$ & $3(60 \%)$ & $2(40 \%)$ & 5 & $4,7 \%$ & 48.258 & 10,36 \\
\hline Total & $46(45 \%)$ & $57(55 \%)$ & $107 * * *$ & $100,0 \%$ & 503.327 & 21,26 \\
\hline
\end{tabular}

*Popolazione stimata per fascia d'età, indipendentemente dal sesso, per il comune di Macapá; ${ }^{* *}$ Coefficiente $(C C S)=$ Totale $n \times 100.000$ / Popolazione stimata per età; Un caso femminile ha fatto ignorare l'elemento "Età".[/caption]

RC: 71075

Disponibile in: https://www.nucleodoconhecimento.com.br/salute/del-covid-19-amacapa 
Tabella 2 Frequenza e percentuale di morbilità precedenti di casi sospetti di COVID19 analizzati. Macapá, Amapá, 13-21 marzo 2020.

Tabela 2 Frequência e porcentagem das morbidades prévias dos casos suspeitos de COVID-19 analisados. Macapá, Amapá, 13 a 21 de Março de 2020.

\begin{tabular}{|c|c|c|c|c|c|c|}
\hline \multirow{2}{*}{ Morbidade Prévia } & \multicolumn{2}{|c|}{ Masculino } & \multicolumn{2}{|c|}{ Feminino } & \multicolumn{2}{|c|}{ Total } \\
\hline & $\mathbf{n}$ & $\%$ & $\mathbf{n}$ & $\%$ & $\mathbf{n}$ & $\%$ \\
\hline $\begin{array}{l}\text { Doença cardiovascular, } \\
\text { incluindo hipertensão }\end{array}$ & 5 & $38,5 \%$ & 8 & $61,5 \%$ & 13 & $100,0 \%$ \\
\hline Doença pulmonar crônica & 3 & $50,0 \%$ & 3 & $50,0 \%$ & 6 & $100,0 \%$ \\
\hline Diabetes & 3 & $60,0 \%$ & 2 & $40,0 \%$ & 5 & $100,0 \%$ \\
\hline Doença hepática & 0 & $0,0 \%$ & 1 & $100,0 \%$ & 1 & $100,0 \%$ \\
\hline Neoplasia & 1 & $100,0 \%$ & 0 & $0,0 \%$ & 1 & $100,0 \%$ \\
\hline Imunodeficiência & 1 & $100,0 \%$ & 0 & $0,0 \%$ & 1 & $100,0 \%$ \\
\hline Doença neurologica crônica & 1 & $100,0 \%$ & 0 & $0,0 \%$ & 1 & $100,0 \%$ \\
\hline $\begin{array}{l}\text { Doença hepática e Doença } \\
\text { neuromuscular e Diabetes }\end{array}$ & 0 & $0,0 \%$ & 1 & $100,0 \%$ & 1 & $100,0 \%$ \\
\hline $\begin{array}{l}\text { Doença hepática e Doença } \\
\text { renal }\end{array}$ & 1 & $100,0 \%$ & 0 & $0,0 \%$ & 1 & $100,0 \%$ \\
\hline Total & 15 & $50,0 \%$ & 15 & $50,0 \%$ & 30 & $100,0 \%$ \\
\hline
\end{tabular}

RC: 71075

Disponibile in: https://www.nucleodoconhecimento.com.br/salute/del-covid-19-amacapa 
Tabella 3 Risultati dei test statistici dei casi sospetti di COVID-19 analizzati. Macapá, Amapá, 13-21 marzo 2020.

\begin{tabular}{|c|c|c|c|c|c|c|c|c|c|c|}
\hline & \multicolumn{6}{|c|}{ Sexo } & \multicolumn{4}{|c|}{ p-valor Testes Estatísticos } \\
\hline \multirow{2}{*}{ Variável } & \multicolumn{3}{|c|}{ Masculino } & \multicolumn{3}{|c|}{ Feminino } & \multirow{2}{*}{$\begin{array}{c}\text { p-valor } \\
\text { normalidade } \\
\text { (Masc./Fem.) }\end{array}$} & \multirow{2}{*}{$\begin{array}{l}\mathrm{p} \text {-valor } \\
\text { teste de } \\
\text { Levene }\end{array}$} & \multirow{2}{*}{$\begin{array}{l}\text { p-valor } \\
\text { teste } t \text { de } \\
\text { Student }\end{array}$} & \multirow{2}{*}{$\begin{array}{l}\text { p-valor U } \\
\text { Mann- } \\
\text { Whitney }\end{array}$} \\
\hline & Média $\pm \mathrm{DP}$ & Mediana & Máx/Min. & Média $\pm \mathrm{DP}$ & Mediana & Máx/Min. & & & & \\
\hline Idade (em anos) & $33,6 \pm 15,0$ & 34,0 & $67 / 0,4$ & $34,6 \pm 14,9$ & 35,5 & $78 / 1$ & $0,64 / 0,51$ & 0,809 & 0,741 & $\ldots$ \\
\hline $\begin{array}{l}\text { Quantitativo de } \\
\text { Sinais/Sintomas } \\
\text { apresentados }\end{array}$ & $5,8 \pm 2,7$ & 5,0 & $12 / 1$ & $6,6 \pm 4,0$ & 5,0 & $20 / 1$ & $0,44 / 0,053$ & 0,037 & -- & 0,488 \\
\hline $\begin{array}{l}\text { Intervalo de dias: } \\
1^{\circ} \text { sinal/sintoma } \\
\text { até data de } \\
\text { notificação }\end{array}$ & $3,4 \pm 2,6$ & 3,0 & $14 / 0$ & $3,5 \pm 3,0$ & 3,0 & $14 / 0$ & $0,00 / 0,004$ & 0,298 & - & 0,941 \\
\hline
\end{tabular}

SD: Deviazione standard; Massimo.: Massimo; Minimo.: Minimo.; Uomini.: Maschio; Fem.: Donna.

Il calcolo del CCS, attraverso la popolazione per fascia d'età nel comune di Macapá, ha mostrato che la fascia d'età di 30-39 anni presentava il più alto CCS, che era di 38,99 casi sospetti ogni 100.000 persone di questa fascia di età. Indipendentemente dall'età, sono stati segnalati 21,26 casi ogni 100.000 persone (tabella 1).

I segni e i sintomi più frequenti tra i casi sospetti analizzati erano tosse, febbre, naso che cola, mal di gola, mal di testa, difficoltà respiratorie, admia, dispnea / aquipnea e mialgia. Così, l' $82,2 \%$ dei pazienti ha avuto tosse, febbre al $68,2 \%$, naso che cola al $58,9 \%$, mal di gola $(57,0 \%)$, mal di testa $(56,1 \%)$, difficoltà respiratorie $(53,3 \%)$, adidamia (36,4\%), dispnea/aquipnea (30,8\%) e mialgia (27,1\%). La differenza di frequenza e percentuale tra i sessi può essere osservata nel grafico $A$ e nel grafico $B$ (figura 1).

RC: 71075

Disponibile in: https://www.nucleodoconhecimento.com.br/salute/del-covid-19-amacapa 
Figura 1 Frequenza e percentuale di segni e sintomi presentati da casi sospetti di COVID-19 analizzati, per genere e generale. Macapá, 13-21 marzo 2020.
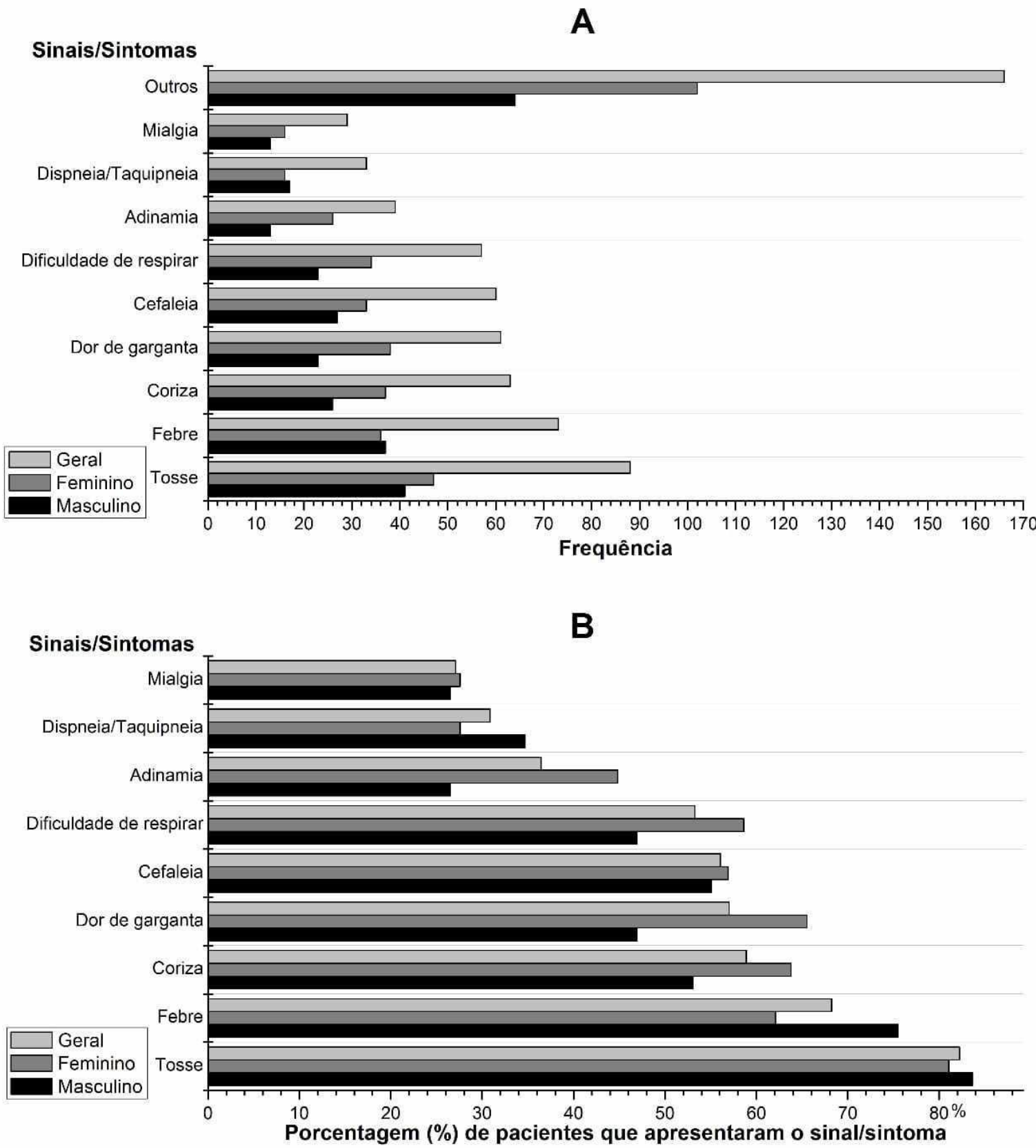

II numero medio di segni e sintomi, per caso sospetto analizzato, è stato $\bar{x}=6,3 \pm 3,5$. Tra i sessi, non vi è stata alcuna differenza statisticamente significativa tra i segni/sintomi quantitativi presentati $(U=1.311 ; p=0,488)$. L'ora, in giorni, tra la data

$\mathrm{RC}: 71075$

Disponibile in: https://www.nucleodoconhecimento.com.br/salute/del-covid-19-amacapa 
del primo segno e sintomo e la data di notifica del caso sospetto era $\bar{x}=3,4 \pm 2,8$, con una mediana di 3 giorni. Non vi è stata alcuna differenza significativa tra i sessi in questo intervallo di tempo $(U=1.221,5 ; p=0,941)$ (tabella 3$)$.

Solo $30(28,04 \%)$ individui, tra i casi sospetti analizzati, hanno presentato morbilità precedenti, dei 107 casi analizzati in questa ite. La morbilità più frequente è stata la "malattia cardiovascolare, compresa l'ipertensione" ( $n=13 ; 43,33 \%)$, seguita da "malattia polmonare cronica" ( $n=6 ; 20,00 \%)$ e "diabete" ( $n=5 ; 16,66 \%)$ (Tabella 2$)$.

L'occupazione più frequente è stata "operatore sanitario" ( $n=9 ; 8,57 \%)$ e 88 (83,81\%) casi, dei 105 analizzati in questo atto, sono stati indicati come "altri". Circa l'81,5\% dei casi è stato segnalato da unità sanitarie pubbliche.

Tra i 108 casi sospetti, 18 persone (16,16\%) 16 (14.81\%) avuto contatti con casi sospetti o confermati di COVID-19 e solo 7 (6,5\%) queste due condizioni sono state esposte. Queste due condizioni sono state ignorate solo in tre dei 108 casi.

\section{ANALISI DELL'INCIDENZA DEI CASI CONFERMATI DI COVID-19 A MACAPÁ E IN ALTRE CAPITALI}

L'incidenza dei casi confermati di COVID-19 a Macapá, fino al 26 marzo 2020, un mese dopo il primo caso confermato in Brasile, è stata di 0,397 casi ogni 100.000 persone. Macapá ha avuto la più bassa incidenza tra le capitali brasiliane. L'incidenza media nelle capitali nazionali $(n=27)$ è stata di $\bar{x}=2,94 \pm 2,19$ e mediana di 2,31. La capitale nazionale con la più alta incidenza è stata Fortaleza, capitale di Ceará, con 8,32 casi ogni 100.000 persone (figura 2).

RC: 71075

Disponibile in: https://www.nucleodoconhecimento.com.br/salute/del-covid-19-amacapa 
Figura 2 Grafico boxplot che rappresenta l'incidenza del COVID-19 nelle capitali brasiliane, per gruppo studiato. Capitali brasiliane, Brasile, dal 26 febbraio 2020 al 26 marzo 2020. (*"outlier", rappresenta la capitale Rio Branco, Acre).

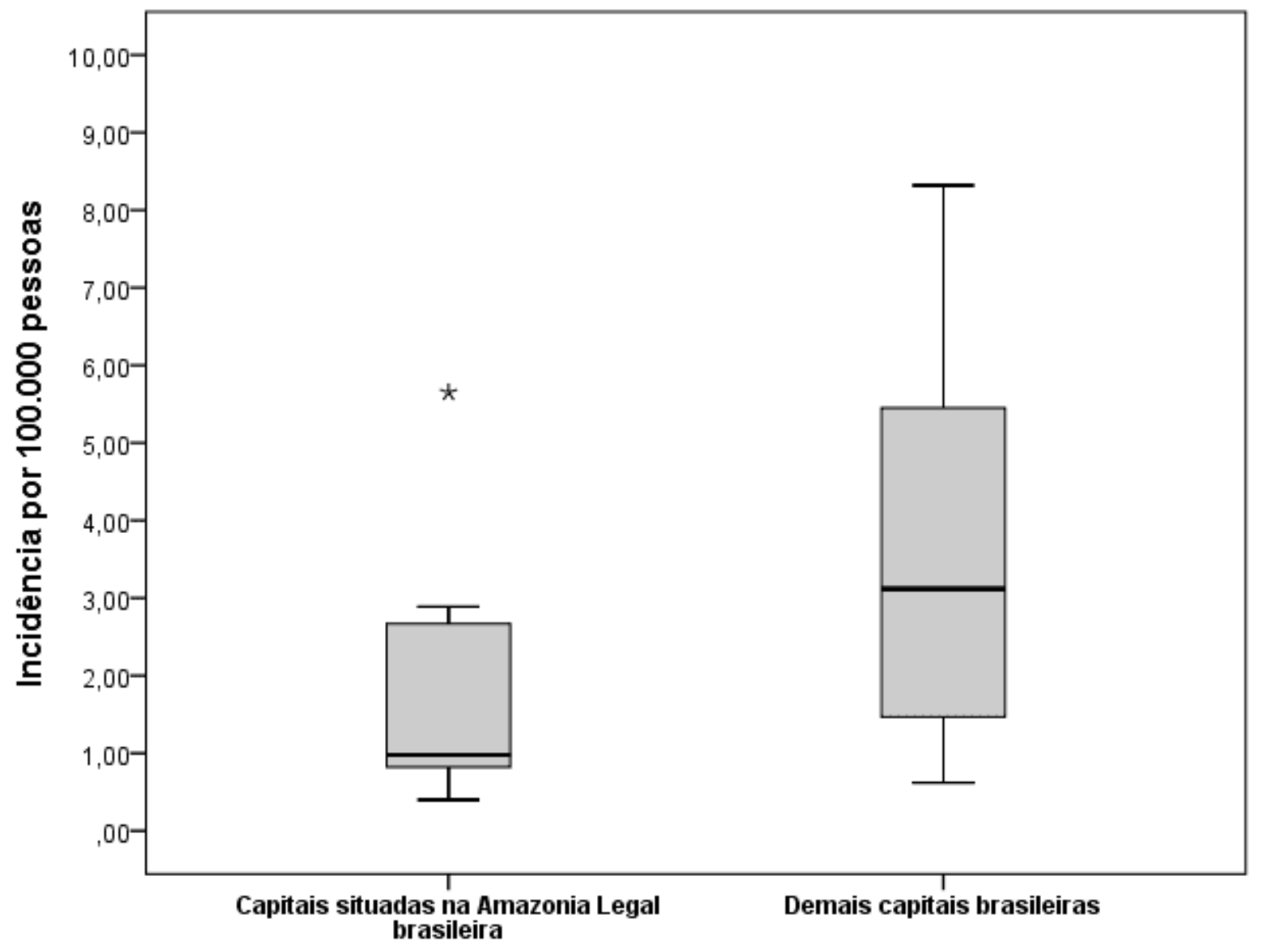

L'incidenza media dei capitali situati nell'Amazzonia legale brasiliana è stata di $\bar{X}=1,86 \pm 1,70$ e mediana di 0,98 . La capitale situata in questa regione, con la più alta incidenza, era Rio Branco, capitale di Acri, con 5,65 casi ogni 100.000 persone (figura 2). C'era una differenza significativa tra l'incidenza di capitali situati nell'Amazzonia legale ( $n=9 ; \bar{x}=1,86 \pm 1,70$; mediana $=0,98)$ e l'incidenza di altri capitali nazionali $(n=18 ; \bar{x}=3,48 \pm 2,24$; mediana $=3,12)(U=41,5 ; p=0,041)$.

RC: 71075

Disponibile in: https://www.nucleodoconhecimento.com.br/salute/del-covid-19-amacapa 
L'incidenza nelle capitali ha mostrato una correlazione positiva e significativa con la densità demografica (DD) dei capoluogi $(r=0,416 ; p=0,031)$, il numero di strutture sanitarie per capitale per capitale $(r=0,539 ; p=0,004)$ e con I'HDI dei capoluogi $(r=0,436 ; \mathrm{p}=0,023)$. E correlazione negativa e significativa con la percentuale di poveri per capitale $(r=-0,409 ; \mathrm{p}=0,034)$ (figura 3 ).

Figura 3 Grafici della correlazione di Pearson tra incidenza e: densità demografica; il numero di strutture sanitarie, l'indice di sviluppo umano e la percentuale di poveri. (MCP: Macapá; $r$ : Coefficiente di correlazione di Pearson).
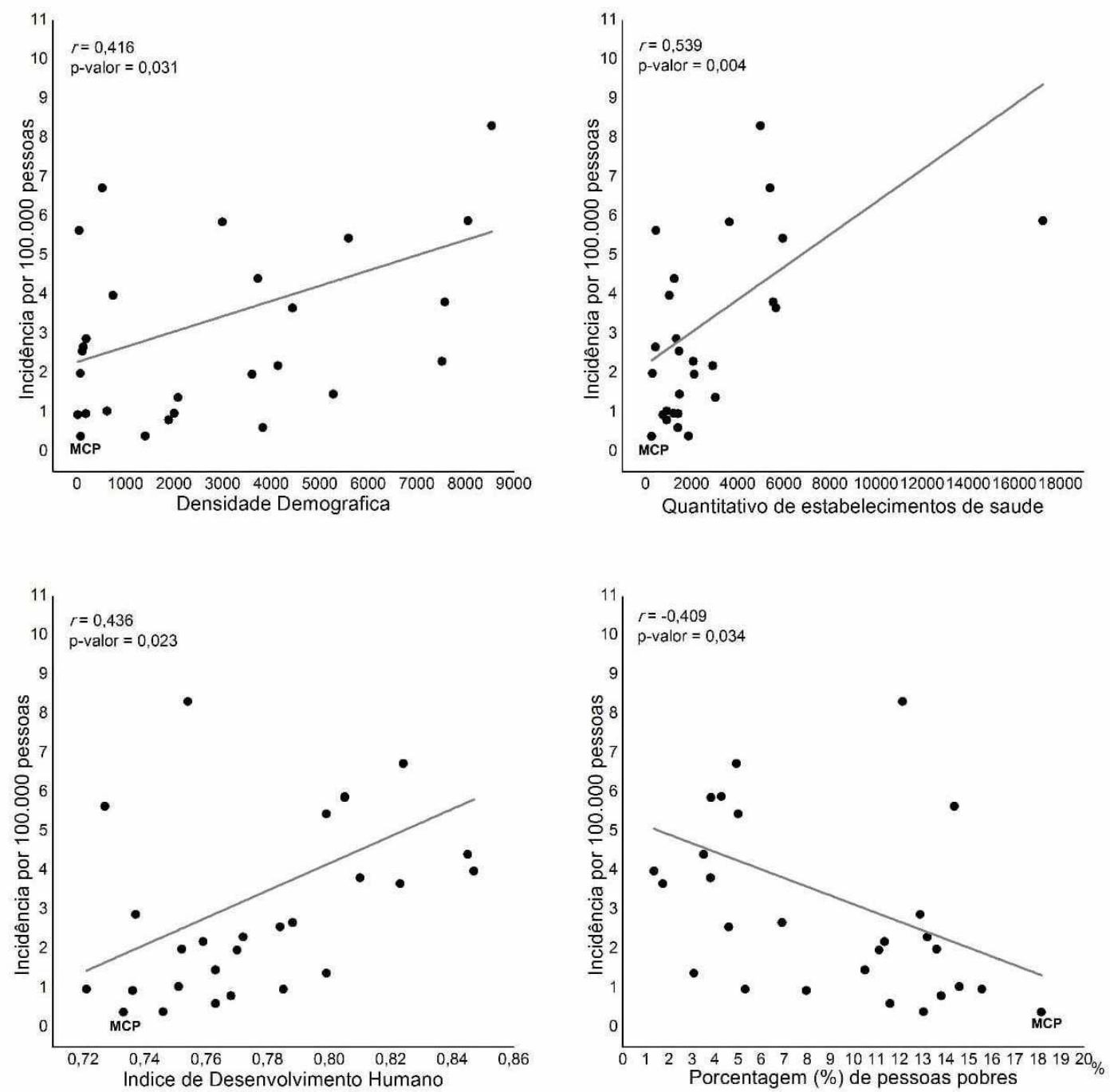

RC: 71075

Disponibile in: https://www.nucleodoconhecimento.com.br/salute/del-covid-19-amacapa 


\section{DISCUSSIONE}

La percentuale di casi confermati ( $n=3 ; 2,78 \%)$, su un totale di 108 segnalati come sospetti nel comune di Macapá, è stata più elevata rispetto ad altri Stati, come Mato Grosso e Minas Gerais, che, entro il 20 marzo 2020, avevano confermato, rispettivamente, 1 (0,81\%) 123 notificati e 38 (0,92\%) casi di 4.122 (SESAMT, 2020; SESAMG, 2020a). II profilo epidemiologico dei tre casi confermati con COVID-19 analizzati nel presente studio è simile al profilo dei primi quattro casi confermati a Minas Gerais, per quanto riguarda la predominanza delle femmine e la storia dei viaggi all'estero in tutti i casi, ma c'è una differenza di circa 10,5 anni nell'età media (SESAMG, 2020b). II numero medio di giorni tra il primo segno e sintomo e la data di notifica dei tre casi confermati è stato inferiore di 0,4 giorni alla media generale. Ciò riduce i tempi di diagnosi e trattamento. riduce inoltre il tempo in cui il paziente infetto rimane in contatto con altre persone con possibilità di contatto e contagio (OMS, 2020a; ZHANG et al., 2020).

La predominanza delle donne $(n=59 ; 54,62 \%)$, su un totale di 108 casi sospetti a Macapá, analizzato in questo studio, conferma il profilo dei casi sospetti divulgati da SESAMG (2020c) il 3 marzo 2020 (56,0\% femminile), con il profilo dei casi sospetti divulgati da SESAMA (2020) il 19 marzo 2020 (62,1\% femminile) e differisce poco dal profilo dei casi sospetti in Brasile, pubblicato dal Ministero della Salute (MS) il 10 febbraio 2020 (53\% maschio) (BRASIL, 2020b). Questa predominanza può essere giustificata dalla leggera percentuale di donne, in relazione agli uomini, a Macapá (BRASIL, 2018b) e dal fatto che le donne sono il principale pubblico di servizi sanitari in Brasile (LEVORATO et al., 2014).

L'età generale media degli indagati nel presente studio, $\bar{x}=34,2 \pm 14,9$ (mediana=34 anni), è vicina al profilo di Minas Gerais, 33 anni (SESAMG, 2020c). Nello Stato di Ceará, fino al 19 marzo 2020, il 42,7\% degli indagati era di età compresa tra i 20 e i 49 anni (SESACE, 2020). Ci si aspettava la somiglianza tra l'età media dei sessi, usando come riferimento lo studio di ZHANG et al., (2020), che ha descritto una

$\mathrm{RC}: 71075$

Disponibile in: https://www.nucleodoconhecimento.com.br/salute/del-covid-19-amacapa 
proporzione femminile di 0,99 uomini/ 1,0 e una predominanza dell' $89,8 \%$ nella fascia di età 30-79 anni dei casi a Wuhan, in Cina.

La fascia d'età, indipendentemente dal sesso, con il maggior numero di casi sospetti segnalati nel presente studio è stata di 30-39 anni, con il 27,1\% dei casi, molto vicina alla percentuale di $20-29$ (25,2\%). Percentuali simili con pari equilibrio per i casi sospetti sono state descritte anche nel Rapporto Epidemiologico del 19 marzo 2020, nello Stato di Rio Grande do Sul (SESARS, 2020). Questa somiglianza tra la semplice percentuale di fasce d'età dei casi sospetti può essere spiegata dalla distribuzione percentuale della popolazione, per fascia d'età, di Macapá (20$29=18,2 \%$; $30-39=14,5 \%)$ e lo Stato di Rio Grande do Sul $(20-29=14,0 \%$; 30 $39=14,6 \%$ ) (BRASIL, 2018a). E anche perché le persone di età compresa tra i 26 e i 49 anni, in cui sono incluse le due fasce d'età 20-29 e 30-39 anni, sono quelle che più cercano servizi sanitari in Brasile (LEVORATO et al., 2014).

Quando si analizza I'CCS di queste due fasce d'età, 38,99 (30-39 anni) e 29,77 (2029 anni) casi ogni 100.000 persone, calcolati in questo studio, è possibile notare che il numero di notifiche ogni 100.000 persone è superiore del $31,0 \%$ nella fascia di età 30-39 anni rispetto ai 20-29 anni. Questa predominanza dell'intervallo di 30-39 anni può essere spiegata da questa fascia di età è inclusa nella fascia di età che più cerca servizi sanitari in Brasile (26-49 anni), e anche dal possibile rapporto tra la domanda di servizi sanitari e la produttività economica del lavoro. Nello Stato di Amapá, la fascia di età più produttiva, con il maggior numero di lavoratori (occupazione formale), 41.365 (31,3\%), ha 30-39 anni (BRASIL, 2018b; LEVORATO et al., 2014). Anche la domanda e l'accesso alla salute sono influenzati dal reddito. Le persone con una migliore situazione economica cercano di più e hanno un migliore accesso ai servizi sanitari. In Brasile, la situazione economica delle persone di età superiore ai 30 anni è sostanzialmente migliore rispetto alle fasce di età più basse (BRASIL, 2018c; TRAVASSOS et al., 2006).

$\mathrm{RC}: 71075$

Disponibile in: https://www.nucleodoconhecimento.com.br/salute/del-covid-19-amacapa 
I segni e i sintomi predominanti studiati nel presente studio, tosse (82,2\%); febbre $(68,2 \%)$; mal di gola $(57,0 \%)$; dispnea/aquipnea $(30,8 \%)$, sono stati descritti come i segni e i sintomi non specifici più comuni nei casi confermati di COVID-19 (BRASIL, 2020a; LI et al., 2020; WHO, 2020a). C'è stato anche accordo con i segni e i sintomi dei pazienti sospetti descritti da SESARS (2020) e BRASIL (2020b). La maggiore discrepanza è stata osservata nel "mal di gola", che ha presentato il $57,0 \%$ dei casi nell'attuale studio e rispetto al $13,9 \%$ dei 55.924 casi cinesi confermati (OMS, 2020a). Questa differenza può essere dovuta al fatto che solo il 2,78\% dei casi analizzati nel presente studio sono confermati, mentre tutti i casi descritti dall'OMS (2020a) hanno una diagnosi confermata di COVID-19. Un'altra possibilità sarebbe la probabile co-infezione da batteri in alcuni casi (ZHANG et al., 2020).

Nel presente studio, il tempo medio, in giorni, tra la data del primo segno/sintomo, riportata dal paziente, e la data di notifica del caso sospetto è stato di $\bar{x}=3,4 \pm 2,8$. Nel $50 \%$ dei casi segnalati come sospetti, il ritardo fino alla notifica è stato maggiore o uguale a tre giorni (mediana=3). Ciò indica che i pazienti considerati sospetti per COVID-19 sono rimasti sintomatici, in media 3,4 giorni, avendo possibili contatti con altre persone. Secondo I'OMS (2020a) la trasmissione della stragrande maggioranza dei casi avviene da pazienti sintomatici, anche se può verificarsi, raramente, da un paziente asintomatico, come dimostrato da ROTHE et al. (2020).

Le precedenti morbilità presentate dai casi sospetti del presente studio sono simili a quelle descritte per 20.812 pazienti cinesi confermati con COVID-19, nello studio di ZHANG et al. (2020), che ha descritto diabete $(5,3 \%)$, ipertensione $(12,8 \%)$, altre malattie cardiovascolari $(4,2 \%)$ malattie polmonari croniche $(2,4 \%)$ morbilità più associate al COVID-19, con ipertensione e altre malattie cardiovascolari più legate a risultati sfavorevoli.

L'unica categoria professionale menzionata nel presente studio è stata la salute ( $n=9 ; 8,57 \%$ ), che è uno dei principali gruppi a rischio di COVID-19 (GALLASCH et al., 2020; ZHANG et al., 2020). Inoltre, vi è stato un elevato numero di marcature

RC: 71075

Disponibile in: https://www.nucleodoconhecimento.com.br/salute/del-covid-19-amacapa 
come "altre" ( $\mathrm{n}=88 ; 83,82 \%)$. Ciò può compromettere la valutazione di altre possibili professioni a rischio, che possono essere correlate a un rischio più elevato di infezione (KOH, 2020; ZHANG et al., 2020;) La stragrande maggioranza dei casi sospetti analizzati in questo studio sono stati segnalati dal Sistema Sanitario Unificato (SUS), che può dimostrare l'importanza e la sovranità di questo sistema statale, che serve la stragrande maggioranza della popolazione brasiliana in modo integrale e libero (BRASIL, 2020f; VIANA et al., 2009).

Una correlazione significativa dell'incidenza del COVID-19 è stata identificata con alcuni indicatori. Almeno nella fase iniziale, fino al 26 marzo 2020, di diffusione del virus, esiste una correlazione tra i valori di incidenza più elevati del COVID-19 e la popolazione che vive nelle città con buoni indicatori di sviluppo sociale.

Tra la correlazione analizzata con l'associazione positiva, viene evidenziato quello eseguito con densità demografica (DD). II maggior numero di casi di COVID19/100.000 persone è correlato con un maggior numero di persone che occupano la stessa area in una determinata località. È importante sottolineare che l'agglomerato di individui facilita la diffusione del virus, poiché le infezioni nei gruppi familiari, così come negli operatori sanitari, confermano il verificarsi della trasmissione da uomo a uomo, principalmente attraverso uno stretto contatto (READ et al., 2020; BRASIL, 2020i). In Cina, le città più densamente occupate, con il maggior numero di voli giornalieri, sono state le più colpite (LAl et al., 2020; READ et al., 2020). Macapá, una città con la più bassa incidenza evidenziata in questo studio, fino a febbraio 2020, ha avuto comunicazione con voli commerciali giornalieri e diretti solo per Brasília, Distretto Federale e Belém, Pará (BRASIL, 2020j).

Le caratteristiche della popolazione legate al COVID-19 sono ancora poco discusse. Alcuni studi, come quello condotto dall'OMS (2020a), possono giustificare il risultato di questa associazione, in quanto mostrano un tasso più elevato di trasmissibilità nelle regioni con una maggiore concentrazione di popolazione. Inoltre, il Ministero della Salute propone generalmente che alcuni focolai in aree ad alta densità di

RC: 71075

Disponibile in: https://www.nucleodoconhecimento.com.br/salute/del-covid-19-amacapa 
popolazione abbiano maggiori possibilità di diffusione e rappresentino quindi eventi con il potenziale di causare un grande impatto sulla salute pubblica (BRASIL, 2009).

Un altro studio di mappatura e analisi spaziale condotto in Cina ha dimostrato che la distribuzione dei casi di COVID-19 non era casuale. I punti di concentrazione dei casi all'inizio dell'epidemia erano limitati alle aree di maggiore sviluppo economico e densità di popolazione. Questi fattori sono legati al maggior numero di casi perché offrono una maggiore mobilità del pendolo, un maggior numero di viaggi e un maggior numero di strutture sanitarie qualificate per eseguire cure e diagnosi (FAN et al., 2020a; LAl et al., 2020; OLIVEIRA et al., 2019; ZASLAVSKY et al., 2017).

Queste evidenze confermano e giustificano i risultati trovati nel presente studio, perché alcuni indicatori sociodemografici e sanitari hanno mostrato una correlazione positiva e significativa con il tasso di incidenza del COVID-19 tra le capitali, cioè la quantificazione e l'analisi dei casi registrati tra il 26 febbraio e il 26 marzo, in Brasile, ha mostrato che le capitali che avevano dd più alti, indice di sviluppo umano più elevato $(\mathrm{HDI})$ e più strutture sanitarie avevano tassi di incidenza più elevati.

Buoni indicatori sociali possono essere correlati a un maggior numero di popolazioni galleggianti, cioè a quella presente sul territorio, in una data specifica, per un breve periodo di tempo e alla ricerca di diverse attività (BRASIL, 2011). Pertanto, le città con buoni indicatori sociali e che offrono possibilità di turismo e affari possono presentare una maggiore incidenza di infezioni, in particolare quelle trasmesse dall'uomo all'uomo, perché forniscono maggiore attrazione per questa popolazione stagionale non residente, poiché l'individuo che compone questo gruppo è un potenziale vettore per la trasmissione del virus (FAN et al., 2020b). Uno studio di correlazione condotto da FAN et al. (2020b), ha valutato l'associazione tra la popolazione galleggiante quantitativa di Wuhan e il numero di casi confermati di COVID-19, ottenuta di conseguenza un coefficiente di correlazione di 0,84. Ciò indica che quando una regione ha un numero maggiore di persone non residenti, in quella regione appariranno casi più confermati.

RC: 71075

Disponibile in: https://www.nucleodoconhecimento.com.br/salute/del-covid-19-amacapa 
La correlazione negativa $(r=-0,409 ; p=0,034)$ tra l'incidenza del COVID-19 e la percentuale di poveri per capitale ha mostrato che maggiore è il numero di casi/100.000 persone, minore è la percentuale di poveri che vivono nella capitale. Questa constatazione riafferma l'esistenza dell'associazione tra incidenza e HDI, osservata anche nel presente studio.

In un primo momento, poiché un maggior numero di casi infetti esportati influenza direttamente la possibilità di un maggior numero di casi secondari nella comunità, $i$ capitali con la più alta percentuale di poveri e di conseguenza che viaggiano meno possono inizialmente avere un'incidenza inferiore (KUCHARSKI et al., 2020; ROSA, 2006). In generale, questa popolazione ha meno accesso alle risorse educative, di informazione e sanitarie. Questi fattori possono ostacolare il riconoscimento dei segni/sintomi iniziali e causare errori di identificazione covid-19, oltre a limitare l'accesso alle strutture sanitarie e, di conseguenza, portare a una sottosegnalazione delle diagnosi (CAMPELLO et al., 2018).

In accordo con queste osservazioni, i risultati del presente studio hanno mostrato che Macapá è la capitale con la più bassa incidenza di COVID-19 ogni 100.000 persone dopo un mese di conferma del primo caso in Brasile. $E$ che, in linea con questo, ha uno degli IPA più bassi e uno dei DD più bassi tra le capitali, il minor numero di strutture sanitarie del paese tra le capitali, un numero limitato di voli commerciali giornalieri e la più alta partecipazione percentuale di poveri tra tutte le capitali nazionali (ATLAS BRASIL, 2020; BRASIL, 2020g; BRASIL, 2020j).

L'incidenza fino al 26 marzo 2020, nel gruppo di capitali dell'Amazzonia legale brasiliana $(\mathrm{n}=9 ; \bar{x}=1,86 \pm 1,70$; mediana $=0,98)$, era significativamente inferiore $(\mathrm{U}$ $=41,5 ; p=0,041)$ rispetto a quella del gruppo delle altre capitali brasiliane $(n=18 ; \bar{x}$ $=3,48 \pm 2,24$; mediana $=3,12$ ). Questa differenza può essere dovuta all'isolamento socio-spaziale dell'Amazzonia legale, in relazione al resto del Brasile, descritto da VIANA et al. (2009). Un esempio di questo isolamento è la capitale Macapá, in cui vive il 59,5\% della popolazione di Amapá, che non è collegata via terra al resto del

RC: 71075

Disponibile in: https://www.nucleodoconhecimento.com.br/salute/del-covid-19-amacapa 
paese (BRASIL, 2020f; DRUMMOND, 2000) e ha presentato, in questo studio, la più bassa incidenza $(0,397)$ di COVID-19 tra le capitali brasiliane.

Questa differenza nell'incidenza del COVID-19 può, oltre all'isolamento sociospaziale, essere correlata a fattori climatologici e urbani in Amazzonia, come l'elevata umidità relativa e la densità demografica (BRASIL 2020f; SILVA et al., 2013; WANG et al., 2020).

All'inizio, i grandi centri urbani dell'Amazzonia brasiliana hanno una storia di epidemie di malattie virali trasmesse dalle zanzare, come Zika e febbre gialla. Tuttavia, i fattori climatici dell'ecosistema amazzonico che agiscono sostenendo la trasmissione endemica e/o l'emergere di onde epidemiche, fornendo la riproduzione delle zanzare (FARIA et al., 2018; GIOVANETTI et al., 2020), potrebbe sfidare la trasmissione del COVID-19 in Amazzonia.

Studi in vitro di SILVA et al. (2013), di WANG et al. (2020) e KAMPF et al., (2020), l'elevata umidità relativa all'aria è costantemente correlata alla minore vitalità dei virus avvolti che causano malattie respiratorie, come il coronavirus. Ciò può essere dovuto alla minore stabilità virale a temperature più elevate e le goccioline respiratorie rimangono meno tempo sospese nell'aria con alta umidità (WANG et al., 2020). I risultati di OLIVEIROS et al. (2020) ha dimostrato che il numero di casi di COVID-19, in un modello di regressione lineare, ha subito solo il 18,0\% di influenza a causa di questi fattori climatici. Mentre la densità di popolazione, i trasporti urbani, gli aspetti culturali, le politiche di sanità pubblica e le misure di allontanamento influenzano l'82,0\%.

Ciò dimostra che, possibilmente, solo i fattori climatici non saranno sufficienti a contenere l'aumento del numero di casi, e sono necessarie altre misure, come la rimozione sociale, decretata dal governo statale in alcune unità federative dell'Amazzonia legale brasiliana, come Amapá (AP, 2020; RIPAP, 2020). In assenza di vaccini efficaci o antivirali, misure come il ritiro sociale, ha il potenziale per ridurre

$\mathrm{RC}: 71075$

Disponibile in: https://www.nucleodoconhecimento.com.br/salute/del-covid-19-amacapa 
l'eccentricità della curva di incidenza covid-19, appiattindone il picco e diminuendo il sovraccarico acuto nel sistema sanitario. È essenziale che le autorità governative siano a conoscenza delle segnalazioni epidemiologiche e di ciò che la comunità scientifica deve contribuire (HAFFAJEE et al., 2020; PREM et al., 2020; RIPAP, 2020; WU et al., 2020).

\section{CONCLUSIONE}

II profilo dei casi sospetti, descritto nel presente studio, è simile al profilo dei casi sospetti e persino, in alcuni punti, al profilo dei casi confermati nella letteratura scientifica, sebbene il numero di studi sul profilo dei casi sospetti sia attualmente limitato. Località come Macapá, che hanno particolari caratteristiche sociali, geografiche ed economiche, possono rivelare un modello di diffusione del COVID-19, il che rende interessante monitorare continuamente e rigorosamente il profilo epidemiologico dei casi.

Macapá, rispetto ad altre località brasiliane, ha un tasso percentuale migliore rispetto alle opzioni adottate per combattere il virus. Tuttavia, il coordinamento della notifica dei casi può essere ottimizzato mediante una comunicazione efficace tra le varie parti interessate, comprese le istituzioni sanitarie e le società sanitarie professionali.

II ruolo del governo nel sensibilizzare l'opinione pubblica sul COVID19 è fondamentale per ridurre l'onere della malattia. Inoltre, è possibile migliorare la registrazione della professione del caso notificato, tra cui, ad esempio, motoboys, ufficio postale e server di pubblica sicurezza, che probabilmente avranno una maggiore esposizione rispetto ad altri professionisti

Oltre l'80,0\% dei casi analizzati sono stati segnalati da unità di sanità pubblica. Ciò dimostra l'importanza di un solido sistema sanitario statale, che può fare la differenza nel contenere l'epidemia di COVID-19, sia a Macapá che nell'Amazzonia brasiliana e nel resto del paese.

RC: 71075

Disponibile in: https://www.nucleodoconhecimento.com.br/salute/del-covid-19-amacapa 
La correlazione tra l'incidenza del COVID-19 e gli indicatori sociali, sociodemografici e sanitari deduce che le capitali con il maggiore sviluppo economico, demografico e sanitario sono state le più colpite dal coronavirus dopo un mese del primo caso in Brasile. Questo risultato è dovuto principalmente al maggiore flusso di viaggiatori. Pertanto, le misure restrittive della mobilità della popolazione, così come il congedo sociale, possono essere più efficaci se dirette a capitali con un ampio flusso di persone.

Le particolari caratteristiche di Macapá, sottolineato in questo studio, in un primo momento hanno rallentato la trasmissibilità del coronavirus nella capitale amapaense. II fatto che Macapá sia lontano dal principale asse socioeconomico nazionale, non abbia rotte terrestri di comunicazione con altre grandi città e abbia un numero ridotto di voli verso altre città, sembra aver contribuito al leggero tasso di incidenza in questa località, fino al 26 marzo 2020. Macapá è la capitale brasiliana con il minor numero di strutture sanitarie. Ciò solleva certamente interrogativi sulle trasmissioni asintomatiche, poiché le infrastrutture sanitarie pubbliche in questa città devono affrontare alcuni limiti. Tuttavia, il tasso percentuale di diagnosi descritto in questo studio per Macapá rimane superiore a quello di alcuni stati brasiliani con migliori indicatori socioeconomici e sanitari. Probabilmente perché i comuni all'interno hanno un apparato sanitario e amministrativo statale più piccolo. Un apparato statale più grande potrebbe essere in grado di influenzare direttamente le condotta e i protocolli presi nelle unità sanitarie, il che può aumentare l'efficacia delle conduzioni diagnostiche. La formazione degli operatori sanitari che lavorano in molti di questi comuni può anche influenzare l'efficacia della diagnosi.

I fattori geografici sono probabilmente influencer per una minore diffusione del virus in Amazzonia, specialmente nei centri urbani piccoli e medi, come Macapá. Questi fattori, insieme alle misure governative di distanziamento sociale della popolazione, gli investimenti nella prevenzione e nella consapevolezza e nella ricerca/sviluppo scientifico volti a combattere il COVID-19 sono essenziali per ridurre l'impatto acuto

RC: 71075

Disponibile in: https://www.nucleodoconhecimento.com.br/salute/del-covid-19-amacapa 
sul sistema sanitario pubblico, che, nella maggior parte di questi centri urbani, è fragile, a causa della disuguaglianza storica tra le regioni brasiliane, per questo tipo di epidemia.

\section{GRAZIE}

Ringraziamo João Silvestre da Silva-Júnior, phD in Sanità Pubblica presso la Scuola di Sanità Pubblica dell'Università di São Paulo e Virgílio Amaral da Cunha Junior, giornalista laureato presso l'Università Federale di Viçosa.

\section{RIFERIMENTI}

AP. Governo do Estado do Amapá. Decreto n 1.414 de 19 de Março de 2020. Dispõe sobre medidas de restrição de aglomeração de pessoas com a finalidade de reduzir os riscos de transmissão do novo Coronavírus (COVID-19) e adota outras providências. Macapá, AP: 2020.

ATLAS BRASIL. Atlas do Desenvolvimento Humano no Brasil. Disponível em: < http://atlasbrasil.org.br/2013/pt/consulta/ >. Acesso em: 24 março 2020.

BRASIL. Ministério da Saúde. Secretaria de Atenção Primária à Saúde. Protocolo de Manejo Clínico do Coronavírus (Covid-19) na Atenção Primária à Saúde. 1. ed. Brasília, DF: Ministério da Saúde - Secretaria de Atenção Primária à Saúde. 2020a.

- Ministério da Saúde. Secretaria de Vigilância em Saúde. Boletim Epidemiológico 02: Infecção humana pelo Novo Coronavírus (2019-nCov). Brasilia, DF: Ministério da Saúde - Centro de Operações de Emergências em Saúde Pública (COE-nCoV): 2020b.

$\mathrm{RC}: 71075$

Disponibile in: https://www.nucleodoconhecimento.com.br/salute/del-covid-19-amacapa 
- Ministério da Saúde. Secretaria de Atenção Especializada à Saúde. Protocolo de Tratamento do Novo Coronavírus (2019-nCoV). 1. ed. Brasília, DF: Ministério da Saúde - Secretaria de Atenção Especializada à Saúde. 2020c.

- Ministério da Saúde. Coronavírus: 25 mortes e 1.546 casos confirmados. Brasilia, DF: 2020d. Disponível em: < https://www.saude.gov.br/noticias/agencia-saude/46573-coronavirus-25-mortes-e-1546-casos-confirmados >. Acesso em: 23 Março 2020.

Ministério da Saúde. Portaria no 454, de 20 de Março de 2020. Declara, em todo o território nacional, o estado de transmissão comunitária do Coronavírus (Covid-19). Brasília, DF: 2020e. Disponível em: < http://www.in.gov.br/en/web/dou/-/portaria-n-454-de-20-de-marco-de-2020249091587 >. Acesso em: 24 Março 2020.

. Instituto Brasileiro de Geografia e Estatística. IBGE Cidades e Estados. 2020f. Disponível em: < https://www.ibge.gov.br/cidades-e-estados >. Acesso em: 23 Março 2020.

Ministério da Saúde. DATASUS. Cadastro Nacional dos Estabelecimentos de Saúde do Brasil - CNES. Quantidade por Tipo de Prestador segundo Capital. Brasília, DF: 2020g. Disponível em:< https://nam10.safelinks.protection.outlook.com/?url=http\%3A\%2F\%2Ftabnet.datasus .gov.br\%2Fcgi\%2Ftabcgi.exe\%3Fcnes\%2Fcnv\%2Festabbr.def\&amp;data=02\%7C01 \%7C\%7C5789bcef877546b7609e08d7d5ac6237\%7C84df9e7fe9f640afb435aaaaaa aaaaaa\%7C1\%7C0\%7C637212808662483071\&amp;sdata=AZP0PC\%2BzxxMGbM TJLdlJO0zLGtXCF9F3BthmFJnvqgo\%3D\&amp;reserved=0 >. Acesso em 24 Março 2020.

Ministério da Saúde. Secretaria de Vigilância em Saúde. Boletim Epidemiológico 05: Doença pelo Coronavírus 2019. Brasilia, DF: Ministério da

RC: 71075

Disponibile in: https://www.nucleodoconhecimento.com.br/salute/del-covid-19-amacapa 
Saúde - Centro de Operações de Emergências em Saúde Pública (COE-nCoV): $2020 \mathrm{~h}$.

. Ministério da Saúde. Sobre a doença. Brasília, DF: 2020i. Disponível em: < https://coronavirus.saude.gov.br/sobre-a-doenca >. Acesso em: 03 Abr. 2020.

Agência Nacional de Aviação Civil. Consulta Interativa - Indicadores do Mercado de Transporte Aéreo. Brasília, DF: 2020j. Disponível em: < https://www.anac.gov.br/assuntos/dados-e-estatisticas/mercado-de-transporteaereo/consulta-interativa/demanda-e-oferta-origem-destino >. Acesso em: 23 Mar. 2020

Instituto Brasileiro de Geografia e Estatística. Pesquisa Nacional por Amostra de Domicílios Contínua - PNAD Contínua 2016-2018, Características Gerais dos Moradores. Brasília, DF: 2018a. Disponível em: < https://www.ibge.gov.br/estatisticas/sociais/populacao/17270-pnadcontinua.html?edicao=24437\&t=downloads >. Acesso em: 24 Março 2020.

. Ministério da Economia. Relação Anual de Informações Sociais: Amapá 2018. Brasília, DF: 2018b. Disponível em: < http://pdet.mte.gov.br/rais?view=default >. Acesso em: 03 Abril 2020.

Instituto Brasileiro de Geografia e Estatística. Coordenação de População e Indicadores Sociais. Síntese de Indicadores Sociais 2018: Uma Análise das Condições de Vida da População Brasileira. Brasília, DF: 2018c.

. Instituto Brasileiro de Geografia e Estatística. Amazônia Legal: Municípios da Amazônia Legal. Brasília, DF: 2014. Disponível em: < https://www.ibge.gov.br/geociencias/informacoes-ambientais/geologia/15819amazonia-legal.html?=\&t=acesso-ao-produto >. Acesso em: 24 Março 2020.

RC: 71075

Disponibile in: https://www.nucleodoconhecimento.com.br/salute/del-covid-19-amacapa 
. Instituto Brasileiro de Geografia e Estatística - IBGE. Atlas de Saneamento

Glossário. Brasília., DF: 2011. Disponível em: https://biblioteca.ibge.gov.br/visualizacao/livros/liv53096_glossario_equipetec.pdf. Acesso em: 29 Mar. 2020.

. Ministério da Saúde. Guia de Vigilância Epidemiológica. Brasília, DF:. 2009. Disponível em: https://bvsms.saude.gov.br/bvs/publicacoes/guia_vigilancia_epidemiologica_7ed.pdf. Acesso em: 01 abr. 2020.

CAMPELLO, T.; GENTILI, P.; RODRIGUES, M.; HOEWELL, G.R. Faces da desigualdade no Brasil: um olhar sobre os que ficam para trás. Saúde em Debate. v.42 n. especial p.54-66, 2018. https://doi.org/10.1590/0103-11042018S305

CASCELLA, M.; RAJNIK, M.; CUOMO, A.; DULEBOHN, S.; DI NAPOLI, R. Features, Evaluation and Treatment Coronavirus (COVID-19). [Atualizado em 8 março 2020] Treasure Island, FL: StatPearls Publishing; 2020. Disponível em: <https://www.ncbi.nlm.nih.gov/books/NBK554776/ > Acesso em: 23 Março 2020.

DOREMALEN, N.V. et al. [Correspondece]. Aerosol and Surface Stability of SARSCoV-2 as Compared with SARS-CoV-1. Destinatário: The New England Journal of $\begin{array}{lllll}\text { Medicine. } & \text { Massachusetts, } & 17 & \text { de }\end{array}$ https://doi.org/10.1056/NEJMc2004973

DRUMMOND, J.A. Investimentos privados, impactos ambientais e qualidade de vida num empreendimento mineral amazônico: o caso da mina de manganês de Serra do Navio (Amapá). História, Ciências, Saúde-Manguinhos. Rio de Janeiro, v. 6, supl. p. 753-792, 2000. https://doi.org/10.1590/S0104-59702000000500002

FAN, J.; LIU, X.; PAN.; DOUGLAS, M.W.; BAO, S. Epidemiology of 2019 Novel Coronavirus Disease-19 in Gansu Province, China, 2020. Emerging Infectious Diseases. v. 26 n.6, 2020a. https://doi.org/10.3201/eid2606.200251

RC: 71075

Disponibile in: https://www.nucleodoconhecimento.com.br/salute/del-covid-19-amacapa 
FAN, C. et al. Prediction of Epidemic Spread of the 2019 Novel Coronavirus Driven by Spring Festival Transportation in China: A Population-Based Study. International Jornal of Envirommental Research and Public Health. v.17 n.5, $2020 \mathrm{~b}$. https://doi.org/10.3390/ijerph17051679

FARIA, N.R. et al. Genomic and epidemiological monitoring of yellow fever virus transmission potential. Science. v.361 n. 6405 p.894-899, 2018. https://doi.org/10.1126/science.aat7115

GALLASCH, C.H.; CUNHA, M.L.; PEREIRA, L.A.S.; SILVA-JUNIOR J.S. Prevenção relacionada à exposição ocupacional do profissional de saúde no cenário de COVID19. Revista Enfermagem UERJ. v. 28 e. 49596. https://doi.org/10.12957/reuerj.2020.49596

GIOVANETTI, M. et al. Genomic and Epidemiological Surveillance of Zika Virus in $\begin{array}{lllll}\text { the Amazon Region. Cell Reports. v.30 n.7, } 2020 . & \end{array}$ https://doi.org/10.1016/j.celrep.2020.01.085

HAFFAJEE, R.L.; MELLO, M.M. Thinking Globally, Acting Locally: The U.S. Response to Covid-19. The New England Journal of Medicine, 2020. https://doi.org/10.1056/NEJMp2006740

KAMPF, G.; TODT, D.; PFAENDER, S.; STEINMANN, E. Persistence of coronaviruses on inanimate surfaces and their inactivation with biocidal agents. The Journal of Hospital Infection. v. 104, n. 3, p. 246-251, 2020. https://doi.org/10.1016/j.jhin.2020.01.022

KUCHARSKI, A.J. et al. Early dynamics of transmission and control of COVID-19: a mathematical modelling study. The Lancet: Infectous Diseases. 11 Março 2020. https://doi.org/10.1016/S1473-3099(20)30144-4

RC: 71075

Disponibile in: https://www.nucleodoconhecimento.com.br/salute/del-covid-19-amacapa 
$\mathrm{KOH}$, D. Occupational risks for COVID-19 infection. Occupational Medicine. v.70 n.1 p. 3-5, 2020. https://doi.org/10.1093/occmed/kqaa036

LAI, S.; BOGOCH, I.I.; WALTS, E.; KHAN, K.; LI, Z.; TATEM, A. Preliminary risk analysis of 2019 novel coronavirus spread within nad beyond China. World pop. No prelo, 2020. Disponível em: < https://www.worldpop.org/resources/docs/china/WorldPop-coronavirus-spread-riskanalysis-v2.pdf >. Acesso: 02 Abril 2020.

LEVIN J.; FOX J.A.; FORDE D.R. Elementary statistics in social research. 12⿺ ed. Upper Saddle River: Pearson Education; 2014.

LEVORATO, C.D.; MELLO, L.M.; SILVA, A.S.; NUNES A.A. Fatores associados à procura por serviços de saúde numa perspectiva relacional de gênero. Ciência e Saúde Coletiva. v. 19 n.4 $\quad$ p. 1263-74, 2020. https://doi.org/10.1590/141381232014194.01242013

LIPSITCH, M.; PHIL, D.; SWERDLOW, D.L.; FINELLI, L. Defining the Epidemiology of Covid-19 - Studies Needed. The New England Journal of Medicine. 2020. https://doi.org/10.1056/NEJMp2002125

LI, W. et al. Angiotensin-converting enzyme 2 is a functional receptor for the SARS coronavirus. Nature. v. 426, 2003. https://www.nature.com/articles/nature02145.pdf

LI, Y.C.; BAI W.Z.; HASHIKAWA, T. The neuroinvasive potential of SARS-CoV2 may be at least partially responsible for the respiratory failure of COVID-19 patients. Journal of Medical Virology. p. 1-4, 2020. https://doi.org/10.1002/jmv.25728

MAPA DO CORONA VÍRUS. POLATO, A.; CUNHA, R.; SORANO, V. (Coord.). Casos de coronavírus no Brasil e no Mundo: mapa e evolução. Disponível em: < https://especiais.g1.globo.com/bemestar/coronavirus/mapa-

RC: 71075

Disponibile in: https://www.nucleodoconhecimento.com.br/salute/del-covid-19-amacapa 
coronavirus/?_ga=2.48817062.1083509909.1584787216-

1227695381.1584787197\#/.> Acesso em: 26 mar. 2020.

MCINTOSH, K. UpToDate. Coronavirus Disease 2019 (COVID-19). USA: 2020. Disponível em: < https://www.uptodate.com/contents/coronavirus-disease-2019covid-19 >. Acesso em: 23 março 2020.

NASSIRI, R. Perspective on Wuhan Viral Pneumonia. Advances in Public Health, Community and Tropical Medicine, V. 02, 2020.

OLIVEIRA, N.M.; PIFFER, M.; STRASSBURG, U. O Indicador de Desenvolvimento Regional no Território do Tocantins. Interações. v.20 n.1 p. 3-20, 2019. http://dx.doi.org/10.20435/inter.v0i0.1607

OLIVEIROS, B.; CARAMELO, L.; FERREIRA, N.C.; CARAMELO, F. Role of temperature and humidity in the modulation of the doubling time of COVID-19 cases. Medrxiv. 2020. https://doi.org/10.1101/2020.03.05.20031872

PREM, K. et al. The effect of control strategies to reduce social mixing on outcomes of the COVID-19 epidemic in Wuhan, China: a modelling study. The Lancet. 25 Março 2020. https://doi.org/10.1016/S2468-2667(20)30073-6

READ, J.M.; BRIDGEN, J.R.E.; CUMMINGS, D.A.T.; HO, A.; JEWELL, C.P. Novel coronavirus 2019-nCoV: early estimation of epidemiological parameters and epidemic predictions. Medrxiv. No prelo, 2020. https://doi.org/10.1101/2020.01.23.20018549

RIPAP. Rede Integrada de Pesquisa do Amapá. Carta Manifesto da Comunidade Científica Amapaense. Macapá. 2020. Disponível em: < https://drive.google.com/file/d/1Kgsikv5B27v60QPyRR6gpyEVjmnVOVfF/view >. Acesso em: 03 abr. 2020.

RC: 71075

Disponibile in: https://www.nucleodoconhecimento.com.br/salute/del-covid-19-amacapa 
ROSA, S.J. Transporte e Exclusão Social: A Mobilidade da População de Baixa Renda da Região Metropolitana de São Paulo e Trem Metropolitano. 2006. Dissertação (Mestrado em Engenharia) - Escola Politécnica da Universidade de São Paulo, São Paulo, 2006. Disponível em: https://www.teses.usp.br/teses/disponiveis/3/3138/tde-07122006163515/publico/dissertacao_silvio_jose_rosa.pdf. Acesso em: 04 abr. 2020.

ROTHAN, H.A.; BYRAREDDY, S.N. The epidemiology and pathogenesis of coronavirus disease (COVID-19) outbreak. Journal of Autoimmunity. No prelo, 2020. https://doi.org/10.1016/j.jaut.2020.102433

ROTHE, C. et al., [Correspondece]. Transmission of 2019-nCoV Infection from an Asymptomatic Contact in Germany. Destinatário: The New England Journal of Medicine. Massachusetts, 5 de Março, 2020. https://doi.org/10.1056/NEJMc2001468

SESACE. Governo do Estado do Ceará. Secretaria de Saúde. Boletim epidemiológico: Doença pelo novo coronavírus (COVID-19) n 18. Fortaleza, CE: 2020.

SESAMA. Governo do Estado do MaranhãO. Secretaria de Saúde. Nota 9 - SES monitora 205 casos suspeitos de COVID-19. São Luís, MA: 2020.

SESAMT. Governo do Estado do Mato Grosso. Secretaria de Saúde. Secretaria Adjunta de Atenção e Vigilância em Saúde. Nota Informativa 16 COVID-19. Cuiabá, MT: 2020.

SESAMG. Governo do Estado de Minas Gerais. Secretaria de Estado de Saúde de Minas Gerais. Subsecretaria de Vigilância em Saúde. Centro de Operações de Emergência em Saúde Pública. Boletim Informativo Diário de 20/03/2020. Belo Horizonte, MG: 2020a.

RC: 71075

Disponibile in: https://www.nucleodoconhecimento.com.br/salute/del-covid-19-amacapa 
SESAMG. Governo do Estado de Minas Gerais. Secretaria de Estado de Saúde de Minas Gerais. Subsecretaria de Vigilância em Saúde. Centro de Operações de Emergência em Saúde Pública. Boletim Informativo Diário de 14/03/2020. Belo Horizonte, MG: 2020b.

SESAMG. Governo do Estado de Minas Gerais. Secretaria de Estado de Saúde de Minas Gerais. Subsecretaria de Vigilância em Saúde. Centro de Operações de Emergência em Saúde Pública. Informe Epidemiológico n³, de $\mathbf{0 3}$ de Março de 2020. Belo Horizonte, MG: 2020c.

SESARS. Governo do Estado do Rio Grande do Sul. Secretaria de Saúde. Centro Operações de Emergências Rio Grande do Sul. Informe Epidemiológico - COVID19 EM 19/03/2020. Porto Alegre, RS: 2020.

SILVA, D.R.; VIANA, V.P.; MÜLLER, A.M.; LIVI, F.P.; DALCIN, P.T.R. Respiratory viral infections and effects of meteorological parameters and air pollution in adults with respiratory symptoms admitted to the emergency room. Influenza and others respiratory viruses. v. 8 n. 1, 2013. https://doi.org/10.1111/irv.12158

TORMAN, V.B.L.; COSTER, R.; RIBOLDI, J. Normalidade de variáveis: métodos de verificação e comparação de alguns testes não-paramétricos por simulação. Revista HCPA. Porto Alegre, v. 32, n. 2, p. 227-234, 2012.

TRAVASSOS C.; OLIVEIRA E.X.G.; VIACAVA F. Desigualdades geográficas e sociais no acesso aos serviços de saúde no Brasil: 1998 e 2003. Ciência \& Saúde Coletiva. v. 11 n. 4 p. $975-986$, 2006. https://doi.org/10.1590/S141381232006000400019

VELAVAN, T.P.; MEYER, C.G. The COVID-19 epidemic. Tropical Medicine \& International Health. v. 25, n.3, 2020. https://doi.org/10.1111/tmi.13383

RC: 71075

Disponibile in: https://www.nucleodoconhecimento.com.br/salute/del-covid-19-amacapa 
VIANA, A.L.D.; IBAÑEZ, N.; ELIAS, P. E. M. Saúde, desenvolvimento e território. 1 ed. São Paulo: Hucitec; 2009.

WANG, J.; TANG, K.; FENG, K.; LV.W. High Temperature and High Humidity $\begin{array}{lllll}\text { Reduce the Transmission of COVID-19. } & \text { SSRN. }\end{array}$ https://dx.doi.org/10.2139/ssrn.3551767

WHO. Word Health Organization. Report of the WHO-China Joint Mission on Coronavirus Disease 2019 (COVID-19). China: Word Health Organization - The Joint Mission. 2020a. Disponível em: < https://www.who.int/docs/defaultsource/coronaviruse/who-china-joint-mission-on-covid-19---final-report-1100hr28feb2020-11mar-update.pdf?sfvrsn=1a13fda0_2\&download=true >

WHO. World Health Organization. Coronavirus disease 2019 (COVID-19): Situacional Report - 62. Genebra, Switzerland. WHO: 2020b.

WHO. World Health Organization. Coronavirus disease 2019 (COVID-19): Situacional Report - 38. Genebra, Switzerland. WHO: 2020c.

WU, J.T.; LEUNG, K.; LEUNG, G.M.; Nowcasting and forecasting the potential domestic and international spread of the 2019-nCoV outbreak originating in Wuhan, China: a modelling study. The Lancet. v. 395 n. 10225 p. 689-697, 2020. https://doi.org/10.1016/S0140-6736(20)30260-9

ZASLAVSKY, R.; GOULART, B. N. G. Migração pendular e atenção à saúde na região de fronteira. Ciência e Saúde Coletiva. 2017, vol.22, n.12. https://doi.org/10.1590/1413-812320172212.03522016

ZHANG, Y. et al. The Novel Coronavirus Pneumonia Emergency Response Epidemiology Team. The epidemiological characteristics of an outbreak of 2019 novel coronavirus diseases (COVID-19) in China. China CDC Weekly. v. 2, n. x, 2020. https://doi.org/10.3760/cma.j.issn.0254-6450.2020.02.003

$\mathrm{RC}: 71075$

Disponibile in: https://www.nucleodoconhecimento.com.br/salute/del-covid-19-amacapa 
Inviato: Aprile, 2020.

Approvato: Aprile, 2020.

RC: 71075

Disponibile in: https://www.nucleodoconhecimento.com.br/salute/del-covid-19-amacapa 